\title{
Some $q$-supercongruences modulo the square and cube of a cyclotomic polynomial
}

\author{
Victor J. W. Guo ${ }^{1} \cdot$ Michael J. Schlosser ${ }^{2}$ (D) \\ Received: 24 January 2021 / Accepted: 21 May 2021 \\ (c) The Author(s) 2021
}

\begin{abstract}
Two $q$-supercongruences of truncated basic hypergeometric series containing two free parameters are established by employing specific identities for basic hypergeometric series. The results partly extend two $q$-supercongruences that were earlier conjectured by the same authors and involve $q$-supercongruences modulo the square and the cube of a cyclotomic polynomial. One of the newly proved $q$-supercongruences is even conjectured to hold modulo the fourth power of a cyclotomic polynomial.
\end{abstract}

Keywords Basic hypergeometric series $\cdot$ Supercongruences $\cdot q$-congruences $\cdot$ Cyclotomic polynomial · Andrews' transformation · Gasper's summation

Mathematics Subject Classification Primary 33D15 - Secondary 11A07 · 11B65

\section{Introduction}

In 1914, Ramanujan [25] listed a number of representations of $1 / \pi$, including

$$
\sum_{k=0}^{\infty}(6 k+1) \frac{\left(\frac{1}{2}\right)_{k}^{3}}{k !^{3} 4^{k}}=\frac{4}{\pi},
$$

where $(a)_{n}=a(a+1) \cdots(a+n-1)$ denotes the Pochhammer symbol. Ramanujan's formulas gained unprecedented popularity in the 1980's when they were discovered to provide

V. J. W. Guo: The first author was partially supported by the National Natural Science Foundation of China (Grant 11771175). M. J. Schlosser: The second author was partially supported by FWF Austrian Science Fund Grant P32305.

$\varangle$ Michael J. Schlosser

michael.schlosser@univie.ac.at

Victor J. W. Guo

jwguo@hytc.edu.cn

1 School of Mathematics and Statistics, Huaiyin Normal University, Huai' an 223300, Jiangsu, People's Republic of China

2 Fakultät für Mathematik, Universität Wien, Oskar-Morgenstern-Platz 1, 1090 Vienna, Austria 
fast algorithms for calculating decimal digits of $\pi$. See, for instance, the monograph [2] by the Borwein brothers.

In 1997, Van Hamme [29] conjectured 13 intriguing $p$-adic analogues of Ramanujan-type formulas, such as

$$
\sum_{k=0}^{(p-1) / 2}(6 k+1) \frac{\left(\frac{1}{2}\right)_{k}^{3}}{k !^{3} 4^{k}} \equiv p(-1)^{(p-1) / 2} \quad\left(\bmod p^{4}\right),
$$

where $p>3$ is a prime. Van Hamme himself supplied proofs for three of them. Supercongruences like (1.2) are called Ramanujan-type supercongruences (see [33]). The proof of the supercongruence (1.2) was first given by Long [22]. As of today, all of Van Hamme's 13 supercongruences have been confirmed by various techniques (see [24,28]).

In recent years, $q$-congruences and $q$-supercongruences have been established by different authors (see, for example, [5-13,15-21,23,27,30-32,34]). In particular, the present authors [9] proved that, for any odd integer $d \geq 5$,

$$
\sum_{k=0}^{n-1}[2 d k+1] \frac{\left(q ; q^{d}\right)_{k}^{d}}{\left(q^{d} ; q^{d}\right)_{k}^{d}} q^{d(d-3) k / 2} \equiv\left\{\begin{array}{lll}
0 & \left(\bmod \Phi_{n}(q)^{2}\right), & \text { if } n \equiv-1 \quad(\bmod d), \\
0 & \left(\bmod \Phi_{n}(q)^{3}\right), & \text { if } n \equiv-1 / 2 \quad(\bmod d) .
\end{array}\right.
$$

Here and in what follows, we adopt the standard $q$-notation: $[n]=1+q+\cdots+q^{n-1}$ is the $q$-integer; $(a ; q)_{n}=(1-a)(1-a q) \cdots\left(1-a q^{n-1}\right)$ is the $q$-shifted factorial, with the compact notation $\left(a_{1}, a_{2}, \ldots, a_{m} ; q\right)_{n}=\left(a_{1} ; q\right)_{n}\left(a_{2} ; q\right)_{n} \cdots\left(a_{m} ; q\right)_{n}$ used for their products; and $\Phi_{n}(q)$ denotes the $n$-th cyclotomic polynomial in $q$, which may be defined as

$$
\Phi_{n}(q)=\prod_{\substack{1 \leq k \leq n \\ \operatorname{gcd}(k, n)=1}}\left(q-\zeta^{k}\right)
$$

where $\zeta$ is an $n$-th primitive root of unity.

We should point out that the $q$-congruence (1.3) does not hold for $d=3$. The present authors [9] also established the following companion of (1.3): for any odd integer $d \geq 3$ and integer $n>1$,

$$
\sum_{k=0}^{n-1}[2 d k-1] \frac{\left(q^{-1} ; q^{d}\right)_{k}^{d}}{\left(q^{d} ; q^{d}\right)_{k}^{d}} q^{d(d-1) k / 2} \equiv\left\{\begin{array}{lll}
0 & \left(\bmod \Phi_{n}(q)^{2}\right), & \text { if } n \equiv 1 \quad(\bmod d), \\
0 & \left(\bmod \Phi_{n}(q)^{3}\right), & \text { if } n \equiv 1 / 2 \quad(\bmod d) .
\end{array}\right.
$$

They also proposed the following conjectures [9, Conjectures 1 and 2], which are generalizations of (1.3) and (1.4).

Conjecture 1 Let $d \geq 5$ be an odd integer. Then

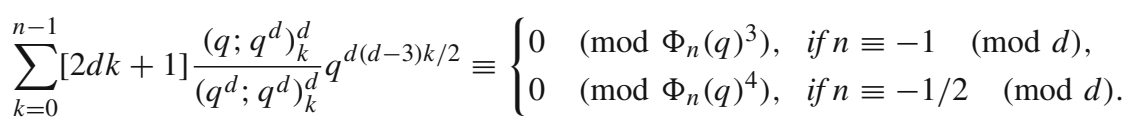

Conjecture 2 Let $d \geq 5$ be an odd integer and let $n>1$. Then

$$
\sum_{k=0}^{n-1}[2 d k-1] \frac{\left(q^{-1} ; q^{d}\right)_{k}^{d}}{\left(q^{d} ; q^{d}\right)_{k}^{d}} q^{d(d-1) k / 2} \equiv\left\{\begin{array}{lll}
0 & \left(\bmod \Phi_{n}(q)^{3}\right), & \text { if } n \equiv 1 \quad(\bmod d), \\
0 & \left(\bmod \Phi_{n}(q)^{4}\right), & \text { if } n \equiv 1 / 2 \quad(\bmod d) .
\end{array}\right.
$$

$q$-Supercongruences such as those above (modulo a third and even fourth power of a cyclotomic polynomial) are rather special. In fact, concrete results for truncated basic hypergeometric sums being congruent to 0 modulo a high power of a cyclotomic polynomial are 
very rare. See $[8,10-12,14,18]$ for recent papers featuring such results. The main goal of this paper is to add two complete two-parameter families of $q$-supercongruences to the list of such $q$-supercongruences (see Theorems 1 and 2).

We shall prove that the respective first cases of Conjectures 1 and 2 are true by establishing the following more general result.

Theorem 1 Let $d$ and $r$ be odd integers satisfying $d \geq 3, r \leq d-4$ (in particular, $r$ may be negative) and $\operatorname{gcd}(d, r)=1$. Let $n$ be an integer such that $n \geq d-r$ and $n \equiv-r(\bmod d)$. Then

$$
\sum_{k=0}^{M}[2 d k+r] \frac{\left(q^{r} ; q^{d}\right)_{k}^{d}}{\left(q^{d} ; q^{d}\right)_{k}^{d}} q^{d(d-r-2) k / 2} \equiv 0 \quad\left(\bmod [n] \Phi_{n}(q)^{2}\right),
$$

where $M=(d n-n-r) / d$ or $n-1$.

We shall also prove the following $q$-supercongruences.

Theorem 2 Let $d$ and $r$ be odd integers satisfying $d \geq 3, r \leq d-4$ (in particular, $r$ may be negative) and $\operatorname{gcd}(d, r)=1$. Let $n$ be an integer such that $n \geq(d-r) / 2$ and $n \equiv-r / 2$ $(\bmod d)$. Then

$$
\sum_{k=0}^{M}[2 d k+r] \frac{\left(q^{r} ; q^{d}\right)_{k}^{d}}{\left(q^{d} ; q^{d}\right)_{k}^{d}} q^{d(d-r-2) k / 2} \equiv 0 \quad\left(\bmod [n] \Phi_{n}(q)\right)
$$

where $M=(d n-2 n-r) / d$ or $n-1$.

The following generalization of the respective second cases of Conjectures 1 and 2 should be true.

Conjecture 3 The q-supercongruence (1.6) holds modulo $[n] \Phi_{n}(q)^{3}$ for $d \geq 5$.

We shall prove Theorems 1 and 2 in Sections 2 and 3, respectively, by making use of Andrews' multiseries extension (2.2) of the Watson transformation [1, Theorem 4], along with Gasper's very-well-poised Karlsson-Minton type summation [3, Eq. (5.13)]. It should be pointed out that Andrews' transformation plays an important part in combinatorics and number theory (see [7] and the introduction of [12] for more such examples).

\section{Proof of Theorem 1}

We need a simple $q$-congruence modulo $\Phi_{n}(q)^{2}$, which was already used in $[10,12]$.

Lemma 1 Let $\alpha, r$ be integers and $n$ a positive integer. Then

$$
\left(q^{r-\alpha n}, q^{r+\alpha n} ; q^{d}\right)_{k} \equiv\left(q^{r} ; q^{d}\right)_{k}^{2} \quad\left(\bmod \Phi_{n}(q)^{2}\right) .
$$


We will further utilize a powerful transformation formula due to Andrews [1, Theorem 4], which may be stated as follows:

$$
\begin{aligned}
& \sum_{k \geq 0} \frac{\left(a, q \sqrt{a},-q \sqrt{a}, b_{1}, c_{1}, \ldots, b_{m}, c_{m}, q^{-N} ; q\right)_{k}}{\left(q, \sqrt{a},-\sqrt{a}, a q / b_{1}, a q / c_{1}, \ldots, a q / b_{m}, a q / c_{m}, a q^{N+1} ; q\right)_{k}}\left(\frac{a^{m} q^{m+N}}{b_{1} c_{1} \cdots b_{m} c_{m}}\right)^{k} \\
& =\frac{\left(a q, a q / b_{m} c_{m} ; q\right)_{N}}{\left(a q / b_{m}, a q / c_{m} ; q\right)_{N}} \sum_{j_{1}, \ldots, j_{m-1} \geq 0} \frac{\left(a q / b_{1} c_{1} ; q\right)_{j_{1}} \cdots\left(a q / b_{m-1} c_{m-1} ; q\right)_{j_{m-1}}}{(q ; q)_{j_{1}} \cdots(q ; q)_{j_{m-1}}} \\
& \times \frac{\left(b_{2}, c_{2} ; q\right)_{j_{1}} \ldots\left(b_{m}, c_{m} ; q\right)_{j_{1}+\cdots+j_{m-1}}}{\left(a q / b_{1}, a q / c_{1} ; q\right)_{j_{1}} \ldots\left(a q / b_{m-1}, a q / c_{m-1} ; q\right)_{j_{1}+\cdots+j_{m-1}}} \\
& \times \frac{\left(q^{-N} ; q\right)_{j_{1}+\cdots+j_{m-1}}}{\left(b_{m} c_{m} q^{-N} / a ; q\right)_{j_{1}+\cdots+j_{m-1}}} \frac{(a q)^{j_{m-2}+\cdots+(m-2) j_{1}} q^{j_{1}+\cdots+j_{m-1}}}{\left(b_{2} c_{2}\right)^{j_{1}} \cdots\left(b_{m-1} c_{m-1}\right)^{j_{1}+\cdots+j_{m-2}}} .
\end{aligned}
$$

This transformation is a multiseries generalization of Watson's ${ }_{8} \phi_{7}$ transformation formula (listed in [4, Appendix (III.18)]; cf. [4, Chapter 1] for the notation of a basic hypergeometric ${ }_{r} \phi_{s}$ series we are using),

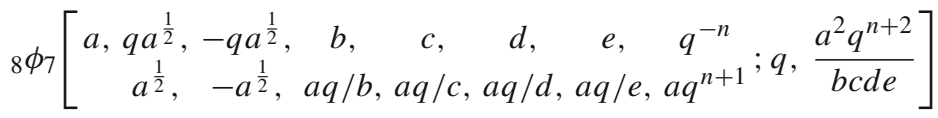

$$
\begin{aligned}
& =\frac{(a q, a q / d e ; q)_{n}}{(a q / d, a q / e ; q)_{n}}{ }_{4} \phi_{3}\left[\begin{array}{c}
a q / b c, d, e, q^{-n} \\
a q / b, a q / c, d e q^{-n} / a
\end{array} ; q, q\right],
\end{aligned}
$$

to which it reduces for $m=2$.

Next, we require a very-well-poised Karlsson-Minton type summation due to Gasper [3, Eq. (5.13)] (see also [4, Ex. 2.33 (i)]):

$$
\begin{gathered}
\sum_{k=0}^{\infty} \frac{\left(a, q \sqrt{a},-q \sqrt{a}, b, a / b, d, e_{1}, a q^{n_{1}+1} / e_{1}, \ldots, e_{m}, a q^{n_{m}+1} / e_{m} ; q\right)_{k}}{\left(q, \sqrt{a},-\sqrt{a}, a q / b, b q, a q / d, a q / e_{1}, e_{1} q^{-n_{1}}, \ldots, a q / e_{m}, e_{m} q^{-n_{m}} ; q\right)_{k}}\left(\frac{q^{1-v}}{d}\right)^{k} \\
=\frac{(q, a q, a q / b d, b q / d ; q)_{\infty}}{(b q, a q / b, a q / d, q / d ; q)_{\infty}} \prod_{j=1}^{m} \frac{\left(a q / b e_{j}, b q / e_{j} ; q\right)_{n_{j}}}{\left(a q / e_{j}, q / e_{j} ; q\right)_{n_{j}}}
\end{gathered}
$$

where $n_{1}, \ldots, n_{m}$ are non-negative integers, $v=n_{1}+\cdots+n_{m}$, and the convergence condition $\left|q^{1-v} / d\right|<1$ is required if the series does not terminate. We point out that an elliptic extension of the terminating $d=q^{-v}$ case of (2.4) can be found in [26, Eq. (1.7)].

In particular, we note that for $d=b q$ the right-hand side of (2.4) vanishes. Putting in addition $b=q^{-N}$ we get the following terminating summation formula:

$$
\sum_{k=0}^{N} \frac{\left(a, q \sqrt{a},-q \sqrt{a}, e_{1}, a q^{n_{1}+1} / e_{1}, \ldots, e_{m}, a q^{n_{m}+1} / e_{m}, q^{-N} ; q\right)_{k}}{\left(q, \sqrt{a},-\sqrt{a}, a q / e_{1}, e_{1} q^{-n_{1}}, \ldots, a q / e_{m}, e_{m} q^{-n_{m}}, a q^{N+1} ; q\right)_{k}} q^{(N-v) k}=0
$$

which is valid for $N>v=n_{1}+\cdots+n_{m}$.

A suitable combination of (2.2) and (2.5) yields the following multi-series summation formula, derived in [12, Lemma 2] (whose proof we nevertheless give here, to make the paper self-contained): 
Lemma 2 Let $m \geq 2$. Let $q$, $a$ and $e_{1}, \ldots, e_{m+1}$ be arbitrary parameters with $e_{m+1}=e_{1}$, and let $n_{1}, \ldots, n_{m}$ and $N$ be non-negative integers such that $N>n_{1}+\cdots+n_{m}$. Then

$$
\begin{aligned}
0= & \sum_{j_{1}, \ldots, j_{m-1} \geq 0} \frac{\left(e_{1} q^{-n_{1}} / e_{2} ; q\right)_{j_{1}} \cdots\left(e_{m-1} q^{-n_{m-1}} / e_{m} ; q\right)_{j_{m-1}}}{(q ; q)_{j_{1}} \cdots(q ; q)_{j_{m-1}}} \\
& \times \frac{\left(a q^{n_{2}+1} / e_{2}, e_{3} ; q\right)_{j_{1}} \ldots\left(a q^{n_{m}+1} / e_{m}, e_{m+1} ; q\right)_{j_{1}+\cdots+j_{m-1}}}{\left(e_{1} q^{-n_{1}}, a q / e_{2} ; q\right)_{j_{1}} \cdots\left(e_{m-1} q^{-n_{m-1}}, a q / e_{m} ; q\right)_{j_{1}+\cdots+j_{m-1}}} \\
& \times \frac{\left(q^{-N} ; q\right)_{j_{1}+\cdots+j_{m-1}}}{\left(e_{1} q^{n_{m}-N+1} / e_{m} ; q\right)_{j_{1}+\cdots+j_{m-1}}} \frac{(a q)^{j_{m-2}+\cdots+(m-2) j_{1}} q^{j_{1}+\cdots+j_{m-1}}}{\left(a q^{n_{2}+1} e_{3} / e_{2}\right)^{j_{1}} \cdots\left(a q^{n_{m-1}+1} e_{m} / e_{m-1}\right)^{j_{1}+\cdots+j_{m-2}}}
\end{aligned}
$$

Proof By specializing the parameters in the multi-sum transformation (2.2) by $b_{i} \mapsto$ $a q^{n_{i}+1} / e_{i}, c_{i} \mapsto e_{i+1}$, for $1 \leq i \leq m$ (where $e_{m+1}=e_{1}$ ), and dividing both sides of the identity by the prefactor of the multi-sum, we obtain that the series on the right-hand side of (2.6) equals

$$
\frac{\left(e_{m} q^{-n_{m}}, a q / e_{1} ; q\right)_{N}}{\left(a q, e_{m} q^{-n_{m}} / e_{1} ; q\right)_{N}} \times \sum_{k=0}^{N} \frac{\left(a, q \sqrt{a},-q \sqrt{a}, e_{1}, a q^{n_{1}+1} / e_{1}, \ldots, e_{m}, a q^{n_{m}+1} / e_{m}, q^{-N} ; q\right)_{k}}{\left(q, \sqrt{a},-\sqrt{a}, a q / e_{1}, e_{1} q^{-n_{1}}, \ldots, a q / e_{m}, e_{m} q^{-n_{m}}, a q^{N+1} ; q\right)_{k}} q^{(N-v) k},
$$

with $v=n_{1}+\cdots+n_{m}$. Now the last sum vanishes by the special case of Gasper's summation stated in (2.5).

Using [11, Lemma 2.1], we can prove the following result which is similar to [11, Lemma 2.2].

Lemma 3 Let $d, n$ be positive integers with $\operatorname{gcd}(d, n)=1$. Let $r$ be an integer. Then

$$
\begin{array}{ll}
\sum_{k=0}^{m}[2 d k+r] \frac{\left(q^{r} ; q^{d}\right)_{k}^{d}}{\left(q^{d} ; q^{d}\right)_{k}^{d}} q^{d(d-r-2) k / 2} \equiv 0 & (\bmod [n]), \\
\sum_{k=0}^{n-1}[2 d k+r] \frac{\left(q^{r} ; q^{d}\right)_{k}^{d}}{\left(q^{d} ; q^{d}\right)_{k}^{d}} q^{d(d-r-2) k / 2} \equiv 0 \quad(\bmod [n]),
\end{array}
$$

where $0 \leq m \leq n-1$ and $d m \equiv-r(\bmod n)$.

We have collected enough ingredients which enables us to prove Theorem 1.

Proof of Theorem 1 The $q$-congruence (1.5) modulo $[n]$ follows from Lemma 3 immediately. In what follows, we shall prove the modulus $\Phi_{n}(q)^{3}$ case of (1.5).

For $M=(d n-n-r) / d$, the left-hand side of (1.5) can be written as the following multiple of a terminating $d+5 \phi_{d+4}$ series:

$[r] \sum_{k=0}^{(d n-n-r) / d} \frac{\left(q^{r}, q^{d+r / 2},-q^{d+r / 2}, q^{r}, \ldots, q^{r}, q^{(d+r) / 2}, q^{d+(d-1) n}, q^{r-(d-1) n} ; q^{d}\right)_{k}}{\left(q^{d}, q^{r / 2},-q^{r / 2}, q^{d}, \ldots, q^{d}, q^{(d+r) / 2}, q^{r-(d-1) n}, q^{d+(d-1) n} ; q^{d}\right)_{k}} q^{d(d-r-2) k / 2}$.

Here, the $q^{r}, \ldots, q^{r}$ in the numerator means $d-1$ instances of $q^{r}$, and similarly, the $q^{d}, \ldots, q^{d}$ in the denominator means $d-1$ instances of $q^{d}$. By Andrews' transformation 
(2.2), we may rewrite the above expression as

$$
\begin{aligned}
& {[r] \frac{\left(q^{d+r}, q^{(r-d) / 2-(d-1) n} ; q^{d}\right)_{(d n-n-r) / d}}{\left(q^{(d+r) / 2}, q^{r-(d-1) n} ; q^{d}\right)_{(d n-n-r) / d}} \sum_{j_{1}, \ldots, j_{m-1} \geq 0} \frac{\left(q^{d-r} ; q^{d}\right)_{j_{1}} \cdots\left(q^{d-r} ; q^{d}\right)_{j_{m-1}}}{\left(q^{d} ; q^{d}\right)_{j_{1}} \cdots\left(q^{d} ; q^{d}\right)_{j_{m-1}}}} \\
& \quad \times \frac{\left(q^{r}, q^{r} ; q^{d}\right)_{j_{1}} \cdots\left(q^{r}, q^{r} ; q^{d}\right)_{j_{1}+\cdots+j_{m-2}}\left(q^{(d+r) / 2}, q^{d+(d-1) n} ; q^{d}\right)_{j_{1}+\cdots+j_{m-1}}}{\left(q^{d}, q^{d} ; q^{d}\right)_{j_{1}} \cdots\left(q^{d}, q^{d} ; q^{d}\right)_{j_{1}+\cdots+j_{m-1}}} \\
& \quad \times \frac{\left(q^{r-(d-1) n} ; q^{d}\right)_{j_{1}+\cdots+j_{m-1}}}{\left(q^{(3 d+r) / 2} ; q^{d}\right)_{j_{1}+\cdots+j_{m-1}}} q^{(d-r)\left(j_{m-2}+\cdots+(m-2) j_{1}\right)+d\left(j_{1}+\cdots+j_{m-1}\right)},
\end{aligned}
$$

where $m=(d+1) / 2$.

It is easy to see that the $q$-shifted factorial $\left(q^{d+r} ; q^{d}\right)(d n-n-r) / d$ contains the factor $1-$ $q^{(d-1) n}$ which is a multiple of $1-q^{n}$. Moreover, since none of $(r-d) / 2,(d+r) / 2$ and $(d+r) / 2+d n-n-r-d$ are multiples of $n$, the $q$-shifted factorials

$$
\left(q^{(r-d) / 2-(d-1) n} ; q^{d}\right)_{(d n-n-r) / d} \text { and }\left(q^{(d+r) / 2} ; q^{d}\right)_{(d n-n-r) / d}
$$

have the same number $(0$ or 1$)$ of factors of the form $1-q^{\alpha n}(\alpha \in \mathbb{Z})$. Besides, the $q$-shifted factorial $\left(q^{r-(d-1) n} ; q^{d}\right)_{(d n-n-r) / d}$ is relatively prime to $\Phi_{n}(q)$. Thus we conclude that the fraction before the multi-sum in (2.7) is congruent to 0 modulo $\Phi_{n}(q)$.

Note that the non-zero terms in the multi-summation in (2.7) are those indexed by $\left(j_{1}, \ldots, j_{m-1}\right)$ that satisfy the inequality $j_{1}+\cdots+j_{m-1} \leq(d n-n-r) / d$ because the factor $\left(q^{r-(d-1) n} ; q^{d}\right)_{j_{1}+\cdots+j_{m-1}}$ appears in the numerator. None of the factors appearing in the denominator of the multi-sum of (2.7) contain a factor of the form $1-q^{\alpha n}$ (and are therefore relatively prime to $\left.\Phi_{n}(q)\right)$, except for $\left(q^{(3 d+r) / 2} ; q^{d}\right)_{j_{1}+\cdots+j_{m-1}}$ when

$$
(d n-d-n-r) /(2 d) \leq j_{1}+\cdots+j_{m-1} \leq(d n-n-r) / d .
$$

Since

$$
\frac{\left(q^{(d+r) / 2} ; q^{d}\right)_{j_{1}+\cdots+j_{m-1}}}{\left(q^{(3 d+r) / 2} ; q^{d}\right)_{j_{1}+\cdots+j_{m-1}}}=\frac{1-q^{(d+r) / 2}}{1-q^{(d+r) / 2+\left(j_{1}+\cdots+j_{m-1}\right) d}},
$$

the denominator of the above fraction contains a factor of the form $1-q^{\alpha n}$ if and only if $j_{1}+\cdots+j_{m-1}=(d n-d-n-r) /(2 d)$ (in this case, the denominator contains the factor $1-q^{(d-1) n / 2}$ ). Writing $n=a d-r$ (with $a \geq 1$ ), we have $j_{1}+\cdots+j_{m-1}=$ $a(d-1) / 2-(r+1) / 2$. Noticing that $m-1=(d-1) / 2$ and $r \leq d-4$, there must exist an $i$ such that $j_{i} \geq a$. Then $\left(q^{d-r} ; q^{d}\right) j_{i}$ has the factor $1-q^{d-r+d(a-1)}=1-q^{n}$ which is divisible by $\Phi_{n}(q)$. Hence the denominator of the reduced form of the multi-sum in (2.7) is relatively prime to $\Phi_{n}(q)$. It remains to show that the multi-sum in (2.7), without the previous fraction, is congruent to 0 modulo $\Phi_{n}(q)^{2}$.

By repeated applications of Lemma 1, the multi-sum in (2.7) (without the previous fraction), modulo $\Phi_{n}(q)^{2}$, is congruent to

$$
\begin{aligned}
\sum_{j_{1}, \ldots, j_{m-1} \geq 0} q^{(d-r)\left(j_{m-2}+\cdots+(m-2) j_{1}\right)+d\left(j_{1}+\cdots+j_{m-1}\right)} \frac{\left(q^{d-r} ; q^{d}\right)_{j_{1}} \cdots\left(q^{d-r} ; q^{d}\right)_{j_{m-1}}}{\left(q^{d} ; q^{d}\right)_{j_{1}} \cdots\left(q^{d} ; q^{d}\right)_{j_{m-1}}} & \\
& \times \frac{\left(q^{r+(m+1) n}, q^{r-(m+1) n} ; q^{d}\right)_{j_{1}} \cdots\left(q^{r+(2 m-2) n}, q^{r-(2 m-2) n} ; q^{d}\right)_{j_{1}+\cdots+j_{m-2}}}{\left(q^{d-m n}, q^{d+m n} ; q^{d}\right)_{j_{1}} \cdots\left(q^{d-(2 m-3) n}, q^{d+(2 m-3) n} ; q^{d}\right)_{j_{1}+\cdots+j_{m-2}}} \\
& \times \frac{\left(q^{d+(d-1) n}, q^{(d+r) / 2} ; q^{d}\right)_{j_{1}+\cdots+j_{m-1}}\left(q^{r-(d-1) n} ; q^{d}\right)_{j_{1}+\cdots+j_{m-1}}}{\left(q^{d-(2 m-2) n}, q^{d+(2 m-2) n} ; q^{d}\right)_{j_{1}+\cdots+j_{m-1}}\left(q^{(3 d+r) / 2} ; q^{d}\right)_{j_{1}+\cdots+j_{m-1}}}
\end{aligned}
$$


where $m=(d+1) / 2$. However, this sum vanishes in light of the $m=(d+1) / 2, q \mapsto q^{d}$, $a=q^{r}, e_{1}=q^{(d+r) / 2}, e_{m}=q^{r-(2 m-2) n}, e_{i}=q^{r-(m+i-2) n}, n_{1}=(d n-d+n+r) /(2 d)$, $n_{m}=0, n_{i}=(n+r-d) / d, 2 \leq i \leq m-1, N=(d n-n-r) / d$ case of Lemma 2. (It is easy to verify that $N-n_{1}-\cdots-n_{m}=d(d-r-2) / 2>0$.) This proves that (1.5) holds modulo $\Phi_{n}(q)^{3}$ for $M=(d n-n-r) / d$.

Since $\left(q^{r} ; q^{d}\right)_{k} /\left(q^{d} ; q^{d}\right)_{k}$ is congruent to 0 modulo $\Phi_{n}(q)$ for $(d n-n-r) / d<k \leq n-1$, we conclude that (1.5) also holds modulo $\Phi_{n}(q)^{3}$ for $M=n-1$.

\section{Proof of Theorem 2}

We first give a simple lemma on a property of certain arithmetic progressions.

Lemma 4 Let $d$ and $r$ be odd integers satisfying $d \geq 3, r \leq d-4$ and $\operatorname{gcd}(d, r)=1$. Let $n$ be an integer such that $n \geq(d-r) / 2$ and $n \equiv-r / 2(\bmod d)$. Then there are no multiples of $n$ in the arithmetic progression

$$
\frac{d+r}{2}, \frac{d+r}{2}+d, \ldots, \frac{d+r}{2}+d n-2 n-r-d .
$$

Proof By the condition $\operatorname{gcd}(d, r)=1$, we have $\operatorname{gcd}((d+r) / 2,(d-r) / 2)=1$. Suppose that

$$
(d+r) / 2+a d=b n
$$

for some integers $a$ and $b$ with $a \geq 0$. Then $(d+r) / 2+a d>(r-d) / 2 \geq-n$ and so $b \geq 0$. Since $n \equiv(d-r) / 2(\bmod d)$, we deduce from (3.2) that $b \equiv-1(\bmod d)$ and thereby $b \geq d-1$. But we have

$$
\frac{d+r}{2}+d n-2 n-r-d=d n-2 n+\frac{d-r}{2}-d \leq(d-1) n-d,
$$

thus implying that no number in the arithmetic progression (3.1) is a multiple of $n$.

Proof of Theorem 2 As before, the $q$-congruence (1.6) modulo $[n]$ can be deduced from Lemma 3. It remains to prove the modulus $\Phi_{n}(q)^{2}$ case of (1.6).

For $M=(d n-2 n-r) / d$, the left-hand side of (1.6) can be written as the following multiple of a terminating $d+5 \phi_{d+4}$ series (this time we changed the position of $q^{(d+r) / 2}$ ):

$$
\begin{aligned}
& {[r] \quad \sum_{k=0}^{(d n-2 n-r) / d} \frac{\left(q^{r}, q^{d+r / 2},-q^{d+r / 2}, q^{(d+r) / 2}, q^{r}, \ldots, q^{r}, q^{d+(d-2) n}, q^{r-(d-2) n} ; q^{d}\right)_{k}}{\left(q^{d}, q^{r / 2},-q^{r / 2}, q^{(d+r) / 2}, q^{d}, \ldots, q^{d}, q^{r-(d-2) n}, q^{d+(d-2) n} ; q^{d}\right)_{k}}} \\
& \quad \times q^{d(d-r-2) k / 2} .
\end{aligned}
$$

Here, the $q^{r}, \ldots, q^{r}$ in the numerator stands for $d-1$ instances of $q^{r}$, and similarly, the $q^{d}, \ldots, q^{d}$ in the denominator stands for $d-1$ instances of $q^{d}$. By Andrews' transformation (2.2), we may rewrite the above expression as

$$
\begin{aligned}
& {[r] \frac{\left(q^{d+r}, q^{-(d-2) n} ; q^{d}\right)_{(d n-2 n-r) / d}}{\left(q^{d}, q^{r-(d-2) n} ; q^{d}\right)_{(d n-2 n-r) / d}} \sum_{j_{1}, \ldots, j_{m-1} \geq 0} \frac{\left(q^{(d-r) / 2} ; q^{d}\right)_{j_{1}}\left(q^{d-r} ; q^{d}\right)_{j_{2}} \cdots\left(q^{d-r} ; q^{d}\right)_{j_{m-1}}}{\left(q^{d} ; q^{d}\right)_{j_{1}}\left(q^{d} ; q^{d}\right)_{j_{2}} \cdots\left(q^{d} ; q^{d}\right)_{j_{m-1}}}} \\
& \quad \times \frac{\left(q^{r}, q^{r} ; q^{d}\right)_{j_{1}} \ldots\left(q^{r}, q^{r} ; q^{d}\right)_{j_{1}+\cdots+j_{m-2}}\left(q^{r}, q^{d+(d-2) n} ; q^{d}\right)_{j_{1}+\cdots+j_{m-1}}}{\left(q^{(d+r) / 2}, q^{d} ; q^{d}\right)_{j_{1}}\left(q^{d}, q^{d} ; q^{d}\right)_{j_{1}+j_{2}} \cdots\left(q^{d}, q^{d} ; q^{d}\right)_{j_{1}+\cdots+j_{m-1}}} \\
& \quad \times \frac{\left(q^{r-(d-2) n} ; q^{d}\right)_{j_{1}+\cdots+j_{m-1}}}{\left(q^{d+r} ; q^{d}\right)_{j_{1}+\cdots+j_{m-1}}} q^{(d-r)\left(j_{m-2}+\cdots+(m-2) j_{1}\right)+d\left(j_{1}+\cdots+j_{m-1}\right)},
\end{aligned}
$$


where $m=(d+1) / 2$.

It is easily seen that the $q$-shifted factorial $\left(q^{d+r} ; q^{d}\right)(d n-2 n-r) / d$ has the factor $1-q^{(d-2) n}$ which is a multiple of $1-q^{n}$. Clearly, the $q$-shifted factorial $\left(q^{-(d-2) n} ; q^{d}\right)(d n-2 n-r) / d$ has the factor $1-q^{-(d-1) n}$ (again being a multiple of $1-q^{n}$ ) since $(d n-2 n-r) / d \geq 1$ holds according to the conditions $d \geq 3, r \leq d-4$, and $n \geq(d-r) / 2$. This indicates that the $q$-factorial $\left(q^{d+r}, q^{-(d-2) n} ; q^{d}\right)_{(d n-2 n-r) / d}$ in the numerator of the fraction before the multi-sum in (3.3) is divisible by $\Phi_{n}(q)^{2}$. Further, it is not difficult to see that the $q$-factorial $\left(q^{d}, q^{r-(d-2) n} ; q^{d}\right)_{(d n-2 n-r) / d}$ in the denominator is relatively prime to $\Phi_{n}(q)$.

Like the proof of Theorem 1, the non-zero terms in the multi-sum in (3.3) are those indexed by $\left(j_{1}, \ldots, j_{m-1}\right)$ satisfying the inequality $j_{1}+\cdots+j_{m-1} \leq(d n-2 n-r) / d$ because of the appearance of the factor $\left(q^{r-(d-2) n} ; q^{d}\right)_{j_{1}+\cdots+j_{m-1}}$ in the numerator. By Lemma 4 , the $q$-shifted factorial $\left(q^{(d+r) / 2}, q^{d}\right)_{j_{1}}$ in the denominator does not contain a factor of the form $1-q^{\alpha n}$ for $j_{1} \leq(d n-2 n-r) / d$ (and are therefore relatively prime to $\Phi_{n}(q)$ ). In addition, none of the other factors appearing in the denominator of the multisum of (3.3) contain a factor of the form $1-q^{\alpha n}$, except for $\left(q^{d+r} ; q^{d}\right)_{j_{1}+\cdots+j_{m-1}}$ when $j_{1}+\cdots+j_{m-1}=(d n-2 n-r) / d$ (in this case the denominator contains the factor $\left.1-q^{(d-2) n}\right)$.

Letting $n=a d+(d-r) / 2($ with $a \geq 0)$, we get $j_{1}+\cdots+j_{m-1}=a(d-2)+(d-r) / 2-1$. If $j_{1} \geq a+1$, then $\left(q^{(d-r) / 2} ; q^{d}\right) j_{1}$ contains the factor $1-q^{(d-r) / 2+a d}=1-q^{n}$. If $j_{1} \leq a$, then $j_{2}+\cdots+j_{m-1} \geq a(d-3)+(d-r) / 2-1$. Since $m-2=(d-3) / 2, d \geq 3$, and $r \leq d-4$, there must be an $i$ with $2 \leq i \leq m-1$ and $j_{i} \geq 2 a+1$. Then $\left(q^{d-r} ; q^{d}\right) j_{i}$ contains the factor $1-q^{d-r+2 a d}=1-q^{2 n}$ which is a multiple of $\Phi_{n}(q)$. Therefore, the denominator of the reduced form of the multi-sum in (3.3) is relatively prime to $\Phi_{n}(q)$. This proves that (3.3) is congruent to 0 modulo $\Phi_{n}(q)^{2}$.

For $M=n-1$, since $\left(q^{r} ; q^{d}\right)_{k} /\left(q^{d} ; q^{d}\right)_{k}$ is congruent to 0 modulo $\Phi_{n}(q)$ for $(d n-$ $2 n-r) / d<k \leq n-1$, we conclude that (1.6) is also true modulo $\Phi_{n}(q)^{2}$ in this case.

Funding Open access funding provided by Austrian Science Fund (FWF).

Open Access This article is licensed under a Creative Commons Attribution 4.0 International License, which permits use, sharing, adaptation, distribution and reproduction in any medium or format, as long as you give appropriate credit to the original author(s) and the source, provide a link to the Creative Commons licence, and indicate if changes were made. The images or other third party material in this article are included in the article's Creative Commons licence, unless indicated otherwise in a credit line to the material. If material is not included in the article's Creative Commons licence and your intended use is not permitted by statutory regulation or exceeds the permitted use, you will need to obtain permission directly from the copyright holder. To view a copy of this licence, visit http://creativecommons.org/licenses/by/4.0/.

\section{References}

1. Andrews, G.E.: Problems and prospects for basic hypergeometric functions. In: Askey, R.A. (ed.) Theory and Application for Basic Hypergeometric Functions, Math. Res. Center, Univ. Wisconsin, Publ. No. 35, pp. 191-224. Academic Press, New York (1975)

2. Borwein, J.M., Borwein, P.B.: Pi and the AGM. Canadian Mathematical Society Series of Monographs and Advanced Texts, vol. 4. Wiley, New York (1998)

3. Gasper, G.: Elementary derivations of summation and transformation formulas for $q$-series. In: Ismail, M.E.H., Masson, D.R., Rahman, M. (eds.) Special Functions, $q$-Series and Related Topics, vol. 14, pp. 55-70. American Mathematical Society, Providence (1997). Fields Inst. Commun.

4. Gasper, G., Rahman, M.: Basic Hypergeometric Series, second edition, Encyclopedia of Mathematics and Its Applications 96. Cambridge University Press, Cambridge (2004)

5. Gorodetsky, O.: $q$-Congruences, with applications to supercongruences and the cyclic sieving phenomenon. Int. J. Number Theory 15, 1919-1968 (2019) 
6. Guo, V.J.W.: Proof of some $q$-supercongruences modulo the fourth power of a cyclotomic polynomial. Results Math. 75, 77 (2020)

7. Guo, V.J.W.: Proof of a generalization of the (C.2) supercongruence of Van Hamme. Rev. R. Acad. Cienc. Exactas Fís. Nat. Ser. A Mat. 115, 45 (2021)

8. Guo, V.J.W., Schlosser, M.J.: Proof of a basic hypergeometric supercongruence modulo the fifth power of a cyclotomic polynomial. J. Differ. Equ. Appl. 25(7), 921-929 (2019)

9. Guo, V.J.W., Schlosser, M.J.: Some new $q$-congruences for truncated basic hypergeometric series. Symmetry 11(2), 268 (2019)

10. Guo, V.J.W., Schlosser, M.J.: Some new $q$-congruences for truncated basic hypergeometric series: even powers. Results Math. 75, 1 (2020)

11. Guo, V.J.W., Schlosser, M.J.: A new family of $q$-supercongruences modulo the fourth power of a cyclotomic polynomial. Results Math. 75, 155 (2020)

12. Guo, V.J.W., Schlosser, M.J.: A family of $q$-hypergeometric congruences modulo the fourth power of a cyclotomic polynomial. Israel J. Math. 240, 821-835 (2020)

13. Guo, V.J.W., Schlosser, M.J.: Some $q$-supercongruences from transformation formulas for basic hypergeometric series. Constr. Approx. 53, 155-200 (2021)

14. Guo, V.J.W., Wang, S.-D.: Some congruences involving fourth powers of central $q$-binomial coefficients. Proc. R. Soc. Edinburgh Sect. A 150(3), 1127-1138 (2020)

15. Guo, V.J.W., Zudilin, W.: A q-microscope for supercongruences. Adv. Math. 346, 329-358 (2019)

16. Guo, V.J.W., Zudilin, W.: Dwork-type supercongruences through a creative $q$-microscope. J. Combin. Theory Ser. A 178, 105362 (2021)

17. Li, L.: Some $q$-supercongruences for truncated forms of squares of basic hypergeometric series. J. Differ. Equ. Appl. 27, 16-25 (2021)

18. Li, L., Wang, S.-D.: Proof of a $q$-supercongruence conjectured by Guo and Schlosser. Rev. R. Acad. Cienc. Exactas Fís. Nat. Ser. A Mat. 114, 190 (2020)

19. Liu, J.-C.: On a congruence involving $q$-Catalan numbers. C. R. Math. Acad. Sci. Paris 358, 211-215 (2020)

20. Liu, J.-C., Huang, Z.-Y.: A truncated identity of Euler and related $q$-congruences. Bull. Aust. Math. Soc. 102, 353-359 (2020)

21. Liu, J.-C., Petrov, F.: Congruences on sums of $q$-binomial coefficients. Adv. Appl. Math. 116, 102003 (2020)

22. Long, L.: Hypergeometric evaluation identities and supercongruences. Pacific J. Math. 249, 405-418 (2011)

23. Ni, H.-X., Pan, H.: Divisibility of some binomial sums. Acta Arith. 194, 367-381 (2020)

24. Osburn, R., Zudilin, W.: On the (K.2) supercongruence of Van Hamme. J. Math. Anal. Appl. 433, 706-711 (2016)

25. Ramanujan, S.: Modular equations and approximations to $\pi$. Quart. J. Math. Oxford Ser. (2) 45, 350-372 (1914)

26. Rosengren, H., Schlosser, M.J.: On Warnaar's elliptic matrix inversion and Karlsson-Minton-type elliptic hypergeometric series. J. Comput. Appl. Math. 178, 377-391 (2005)

27. Straub, A.: Supercongruences for polynomial analogs of the Apéry numbers. Proc. Am. Math. Soc. 147, 1023-1036 (2019)

28. Swisher, H.: On the supercongruence conjectures of van Hamme. Res. Math. Sci. 2, 18 (2015)

29. Van Hamme, L.: Some conjectures concerning partial sums of generalized hypergeometric series. $p$-Adic Functional Analysis, (Nijmegen, 1996), Lecture Notes in Pure and Appl. Math., vol. 192, pp. 223-236. Dekker, New York (1997)

30. Wang, X., Yu, M.: Some new $q$-congruences on double sums. Rev. R. Acad. Cienc. Exactas Fís. Nat., Ser. A Mat. 115, 9 (2021)

31. Wang, X., Yue, M.: Some $q$-supercongruences from Watson's ${ }_{8} \phi_{7}$ transformation formula. Results Math. 75, 71 (2020)

32. Wang, X., Yue, M.: A $q$-analogue of a Dwork-type supercongruence. Bull. Aust. Math. Soc. 103, 303-310 (2021)

33. Zudilin, W.: Ramanujan-type supercongruences. J. Number Theory 129(8), 1848-1857 (2009)

34. Zudilin, W.: Congruences for $q$-binomial coefficients. Ann. Combin. 23, 1123-1135 (2019)

Publisher's Note Springer Nature remains neutral with regard to jurisdictional claims in published maps and institutional affiliations. 